\title{
Familial occurrence of autoimmune liver disease with overlapping features of primary biliary cholangitis and autoimmune hepatitis in a mother and her daughter
}

\author{
Kaoru Omori $^{1}$ (D) Kanako Yoshida ${ }^{1} \cdot$ Masaki Yokota $^{2} \cdot$ Tsutomu Daa $^{3}$ • \\ Masahiro Kan ${ }^{1}$
}

Received: 25 March 2016/ Accepted: 22 July 2016/Published online: 8 August 2016

(c) The Author(s) 2016. This article is published with open access at Springerlink.com

\begin{abstract}
We encountered two patients with overlapping features of primary biliary cholangitis and autoimmune hepatitis within the same family. A 68-year-old woman presented at our hospital from a previous medical institution because of the diagnosis of primary biliary cholangitis. Her 49-year-old daughter was admitted with liver dysfunction 4 years later. When compared, these two related patients were found to have overlapping features of primary biliary cholangitis and autoimmune hepatitis. Their human leukocyte antigen haplotype was DRB1*04:05/ DRB1*15:02. The clinical and biochemical findings of these two patients immediately improved following treatment with a combination of prednisolone and ursodeoxycholic acid, in accordance with the Japanese guidelines. It is extremely important to identify such pathological conditions as quickly as possible, particularly with the appearance of severe liver dysfunction due to liver cirrhosis, as observed in our case. The Japanese guidelines are considered to be a realistic and useful clinical policy for the swift and efficient treatment of patients with overlapping features of primary biliary cholangitis and autoimmune hepatitis. We suggest that our two patients presented with a genetic predisposition to autoimmune liver disease with
\end{abstract}

Kaoru Omori

meitokukai-omo@sato-d1.com

1 Department of Gastroenterology and Hepatology, Sato Daiichi Hospital, 77-1 Hokyoji, Usa, Oita 879-0454, Japan

2 Department of Gastroenterology, Nakatsu Municipal Hospital, 173 Shimo-Ikenaga, Nakatsu, Oita 871-8511, Japan

3 Department of Pathology, Graduate School of Medicine, Faculty of Medicine, Oita University, 1-1 Idaigaoka, Hasama-machi, Yufu, Oita 879-5593, Japan overlapping features of primary biliary cholangitis and autoimmune hepatitis within the same family.

Keywords Primary biliary cholangitis · Autoimmune hepatitis · Overlap syndrome $\cdot$ Familial

\section{Introduction}

Primary biliary cholangitis (PBC) and autoimmune hepatitis (AIH) are both considered to be autoimmune diseases of the liver. Although they share the autoimmune aspect, their pathogenesis, clinical features, disease course, and response to therapy are quite distinct. PBC is a slowly progressive disease primarily of the intrahepatic bile ducts, with a strong preponderance of females and a median age of 50 years at diagnosis. However, familial $\mathrm{PBC}$ is rare ( $\sim 1-6 \%$ of PBC cases) and an earlier study suggested that human leukocyte antigen (HLA)-DR8 represents a risk factor for developing PBC [1]. Therapy with ursodeoxycholic acid (UDCA) appears to slow the progression of this disease. On the other hand, AIH is a chronic disorder characterized by continuing hepatocellular necrosis and inflammation, usually with fibrosis, which can evolve into liver cirrhosis and failure. AIH commonly affects young women, who usually exhibit characteristic autoantibodies and a characteristic immunogenetic background involving HLA-B8, DR3, or DR4 [2]. Familial cases of AIH are reported to occur in only $1 \%$ of AIH cases [3]. Moreover, response to immunosuppressive therapy is usually prompt in this disease, resulting in a good long-term prognosis [4].

Patients with overlapping features of $\mathrm{PBC}$ and $\mathrm{AIH}$ have been recognized for some time. Although the prevalence of autoimmune liver disease with overlapping features of $\mathrm{PBC}$ and $\mathrm{AIH}$ has been reported to vary considerably 
(2.1-19\%) [5], there are few reports of cases occurring within the same family. Here we report overlapping features of PBC and $\mathrm{AIH}$ in the mother and daughter of the same family.

\section{Case report}

Case 1: a 68-year-old woman was admitted to a previous medical institution because of liver dysfunction. She was diagnosed with PBC by the following biochemical and serological findings: aspartate aminotransferase (AST) $212 \mathrm{U} / \mathrm{L}$, alanine aminotransferase (ALT) $203 \mathrm{U} / \mathrm{L}$, total bilirubin (T-Bil) $0.9 \mathrm{mg} / \mathrm{dL}$, alkaline phosphatase (ALP) $491 \mathrm{U} / \mathrm{L}, \gamma$-glutamyl transpeptidase $(\gamma$-GTP) $212 \mathrm{U} / \mathrm{L}$, immunoglobulin (Ig) M $534 \mathrm{mg} / \mathrm{dL}$, and anti-mitochondrial antibody (AMA) titer 1:80. Ultrasonography (US) and computed tomography (CT) showed morphological changes indicative of liver cirrhosis. Although the patient was immediately treated with $600 \mathrm{mg}$ UDCA, the response to this treatment was not sufficient. She was referred by another medical institution to our hospital for further, more detailed examinations and was subsequently admitted to our hospital due to further liver dysfunction and progressive symptoms over the previous 4 weeks, including general fatigue, appetite loss, and muscle cramp in her legs. Her initial laboratory tests revealed liver dysfunction with an antinuclear antibody (ANA) titer of 1:320 with a homogeneous pattern, and elevation of $\operatorname{IgG}$ to $3,517 \mathrm{mg} / \mathrm{dL}$ (Table 1). Serological examinations for hepatitis A virus (HAV), hepatitis B virus (HBV), and hepatitis $\mathrm{C}$ virus (HCV) were all negative (Table 1). There was no history of symptoms suggestive of viral infection. International AIH score on the basis of the simplified International Autoimmune Hepatitis Group (IAIHG) scoring system and the revised AIH scoring system was 6 points and 13 points, respectively, implying a probable AIH $[6,7]$. The HLA-DRB1 haplotype was DRB1*04:05/DRB1*15:02. Although the patient had ingested some health foods, liver dysfunction had not improved when these foods were stopped. Drug-induced lymphocyte stimulation tests for such food types were negative. Thus, the patient was eventually diagnosed on the basis of both the Paris criteria and Japanese guidelines with an autoimmune liver disease with overlapping features of PBC and AIH [4, 8]. We immediately administered $5 \mathrm{mg} /$ day prednisolone (PSL) and $600 \mathrm{mg} / \mathrm{day}$ of UDCA [9]. The PSL dose given was smaller than that indicated by the Japanese guidelines because of the complication of liver cirrhosis and the consequent increased risk of opportunistic infections, osteoporosis, and abnormalities in glucose metabolism. This course of treatment resulted in improvements to clinical and biochemical findings within 1 month. Since then, liver function has remained within the normal range, resulting in a constant liver cirrhotic condition of Child-Pugh class A (Fig. 1). Furthermore, the current level of bone mineral density (BMD) is $0.396 \mathrm{~g} / \mathrm{cm}^{2}$ greater than the normal range $\left(0.261-0.333 \mathrm{~g} / \mathrm{cm}^{2}\right)$, although we continue to administer PSL at a dose of $5 \mathrm{mg} /$ day.

Case 2: a 49-year-old daughter was diagnosed with liver dysfunction for the first time during medical examinations. She had a variety of symptoms, including general fatigue and darkness of the urine for 2 weeks previously. She visited our hospital and was admitted because of visible jaundice and laboratory tests indicative of liver dysfunction: ALT $1425 \mathrm{U} / \mathrm{L}$, AST $1203 \mathrm{U} / \mathrm{L}$, ALP $900 \mathrm{U} / \mathrm{L}, \gamma$ GTP $208 \mathrm{U} / \mathrm{L}$, T-Bil $4.79 \mathrm{mg} / \mathrm{dL}$, AMA titer 1:40, a positive AMA-M2 antibody, ANA 1:80 of the speckled type, and elevation of IgG to $2261 \mathrm{mg} / \mathrm{dL}$ (Table 1). Serological examinations for HAV, HBV, and HCV were all negative (Table 1). There was no history of symptoms suggestive of viral infection. The revised AIH score was 14 points, implying a probable AIH. However, the simplified IAIHG score was 7 points, implying a definite AIH $[6,7]$. US and CT ruled out obstructive jaundice. The HLA-DRB1 haplotype was DRB1*04:05/DRB1*15:02, as observed with case 1 . There was no history of medication use. Thus, we eventually diagnosed the patient, on the basis of both the Paris criteria and Japanese guidelines [4, 8], with an autoimmune liver disease with overlapping features of $\mathrm{PBC}$ and $\mathrm{AIH}$. Pathological findings from liver biopsies revealed interface hepatitis with dense portal and periportal lymphocytic/lymphoplasmacytic infiltrations (Fig. 2). We immediately treated the patient with a combination of $600 \mathrm{mg} /$ day UDCA and $30 \mathrm{mg} /$ day PSL in accordance with the Japanese guidelines, which resulted in improvements of both clinical and biochemical findings within 1 month. Since then, liver function has remained normal, the current BMD is $0.508 \mathrm{~g} / \mathrm{cm}^{2}$ greater than the normal range $\left(0.416-0.488 \mathrm{~g} / \mathrm{cm}^{2}\right)$ and PSL has been slowly reduced to $7.5 \mathrm{mg}$ (Fig. 3).

Familial studies (Table 1): the mother (case 1) has two children: the first daughter (case 2) and a second daughter. We tested liver function, autoantibodies, and HLA haplotype in all family members and subsequently compared their clinical backgrounds. The second daughter was healthy and had no history of liver dysfunction. Laboratory findings for the second daughter revealed normal liver function, a negative ANA, and a positive AMA-M2 antibody. The first daughter (case 2) had exactly the same HLA haplotype as the mother (case 1), whereas the second daughter's HLA haplotype was DRB $1 * 04: 05 /$ DRB $1 * 01: 01$. There was no difference between any of the family members with respect to medication use, smoking status, alcohol consumption, or obstetric history. The 
Table 1 Laboratory data at the first medical examination and comparison of environmental and genetic factors between all family members

\begin{tabular}{|c|c|c|c|}
\hline & Case 1 (mother) & Case 2 (daughter 1) & Daughter 2 \\
\hline Age (years) & 68 & 49 & 48 \\
\hline \multicolumn{4}{|l|}{ Biochemistry } \\
\hline $\mathrm{TP}(6.5-8.3)(\mathrm{g} / \mathrm{dL})$ & 7.8 & 8.4 & 7.3 \\
\hline Alb $(3.8-5.3)(\mathrm{g} / \mathrm{dL})$ & 2.9 & 3.9 & 4.4 \\
\hline T.Bil $(0.2-1.2)(\mathrm{mg} / \mathrm{dL})$ & 4.25 & 4.79 & 0.56 \\
\hline AST (5-40) (IU/L) & 328 & 1203 & 13 \\
\hline ALT (3-35) (IU/L) & 312 & 1425 & 10 \\
\hline$\gamma$-GTP (10-60) (IU/L) & 60 & 208 & 20 \\
\hline ALP (100-340) (IU/L) & 418 & 900 & 149 \\
\hline \multicolumn{4}{|l|}{ Hematology } \\
\hline WBC (4000-8000) $(/ \mu \mathrm{L})$ & 3400 & 5100 & 6400 \\
\hline $\mathrm{RBC}(350-480)\left(\times 10^{4} / \mu \mathrm{L}\right)$ & 316 & 446 & 436 \\
\hline $\mathrm{Hb}(11.5-16.0)(\mathrm{g} / \mathrm{dL})$ & 10.8 & 14.7 & 13.8 \\
\hline Plt $(12.0-40.0)\left(\times 10^{4} / \mathrm{uL}\right)$ & 11.8 & 21.1 & 26.6 \\
\hline PT \% (\%) & 41.6 & 79.8 & 94.0 \\
\hline APTT (26-38) (s) & 41.0 & 35.1 & 27.7 \\
\hline \multicolumn{4}{|l|}{ Serology } \\
\hline $\operatorname{IgG}(820-1740)(\mathrm{mg} / \mathrm{dL})$ & 3517 & 2261 & 1249 \\
\hline $\operatorname{IgA}(90-400)(\mathrm{mg} / \mathrm{dL})$ & 370 & 386 & 121 \\
\hline $\operatorname{IgM}(52-270)(\mathrm{mg} / \mathrm{dL})$ & 491 & 203 & 173 \\
\hline ANA (0-79) & $\times 320$ & $\times 80$ & $<40$ \\
\hline AMA (0-19) & $(\times 80)$ & $\times 40$ & Not tested \\
\hline AMA-M2 (0.0-6.9) & 161.9 & 55.8 & 75.7 \\
\hline ASMA (0-19) & $<20$ & $<20$ & Not tested \\
\hline Anti-LKM1 (0-16.9) & $<5.0$ & 5.2 & Not tested \\
\hline \multicolumn{4}{|l|}{ Viral marker } \\
\hline IgM anti-HA & - & - & Not tested \\
\hline HBs-Ag & - & - & - \\
\hline $\mathrm{HCV}-\mathrm{Ab}$ & - & - & - \\
\hline IgM anti-HBc $(0-0.9)$ & $(-)$ & - & Not tested \\
\hline IgG anti-HBc (0-0.99) & $(-)$ & - & Not tested \\
\hline HCV-RNA (PCR) & $(-)$ & - & Not tested \\
\hline HLA-DRB1 haplotype & $\begin{array}{l}\text { DRB1*04:05, } \\
\text { DRB1*15:02 }\end{array}$ & $\begin{array}{l}\text { DRB1*04:05, } \\
\text { DRB1*15:02 }\end{array}$ & $\begin{array}{l}\text { DRB1*04:05, } \\
\text { DRB1*01:01 }\end{array}$ \\
\hline $\begin{array}{l}\text { Smoking status/alcohol } \\
\text { consumption }\end{array}$ & Non-smoker/nil & Non-smoker/nil & Non-smoker/nil \\
\hline \multirow[t]{2}{*}{ Occupation } & Housewife & Nurse & Nurse \\
\hline & 18 years-present & 20 years-present & 20 years-present \\
\hline $\begin{array}{l}\text { Period of time living with } \\
\text { mother }\end{array}$ & & Birth-20 years & Birth-20 years \\
\hline Previous medical history & Unremarkable & Unremarkable & Unremarkable \\
\hline Medication use & Nil & Nil & Nil \\
\hline Obstetric history & 2 deliveries & 3 deliveries & 2 deliveries \\
\hline \multicolumn{4}{|l|}{$\begin{array}{l}\text { Serological evidence of past } \\
\text { infection }\end{array}$} \\
\hline Epstein-barr virus & + & + & Not tested \\
\hline Cytomegalovirus & + & + & Not tested \\
\hline
\end{tabular}

(-) Serological findings of a previous medical institution, ANA antinuclear antibody, AMA anti-mitochondrial antibody, AMA-M2 anti-mitochondrial antibody-M2, ASMA anti-smooth muscle antibody, LKM1 liver-kidney microsome type $1, H L A$ human leukocyte antigen

Normal ranges given in parentheses 
Fig. 1 Clinical course of case 1 (mother). Following diagnosis with an autoimmune liver disease with overlapping features of primary biliary cholangitis and autoimmune hepatitis, the patient was treated with a combination of $600 \mathrm{mg} /$ day ursodeoxycholic acid (UDCA) and $5 \mathrm{mg} /$ day prednisolone $(P S L)$ in accordance with the Japanese guidelines, resulting in the gradual improvement of both clinical and biochemical findings. Key: $A N A$ antinuclear antibody, $A M A-M 2$ antimitochondrial antibody-M2

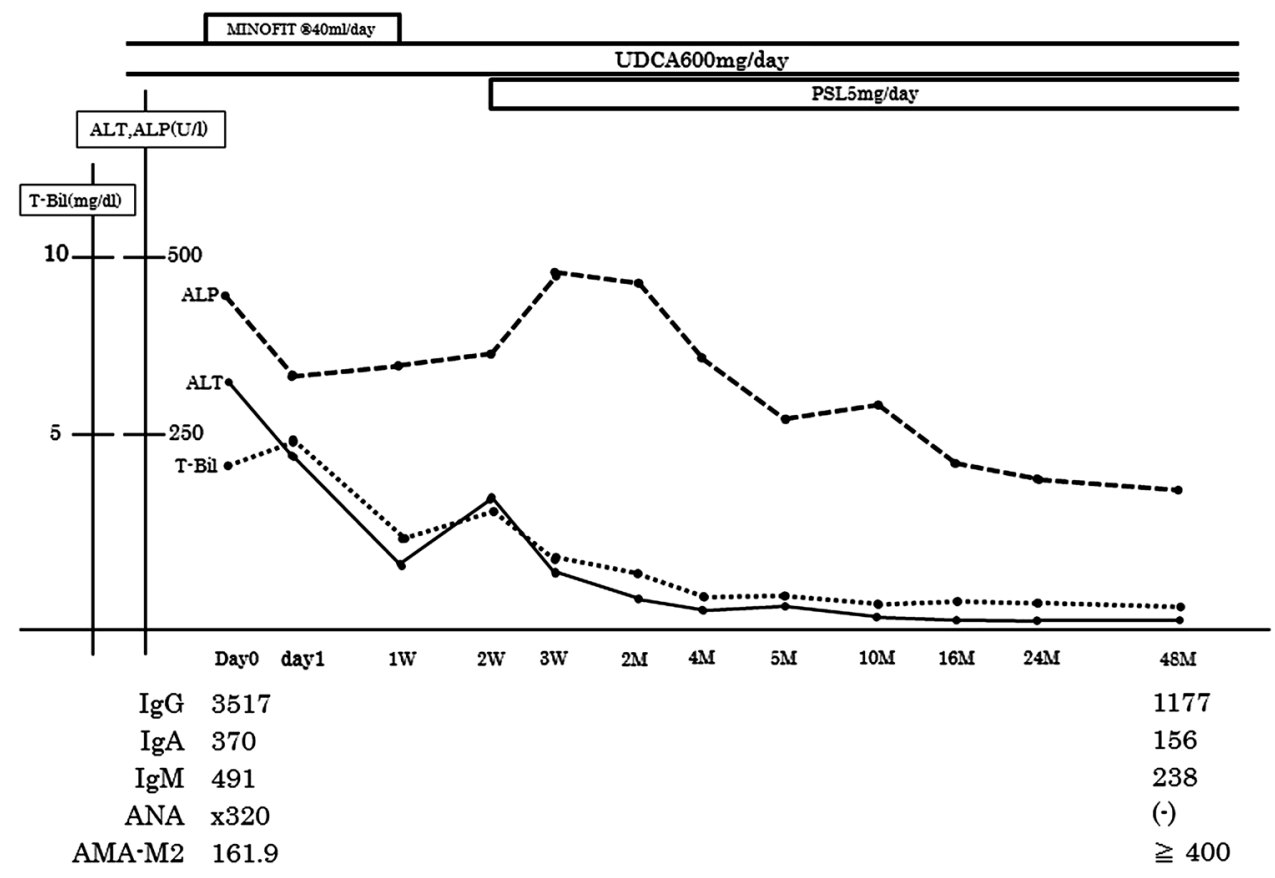

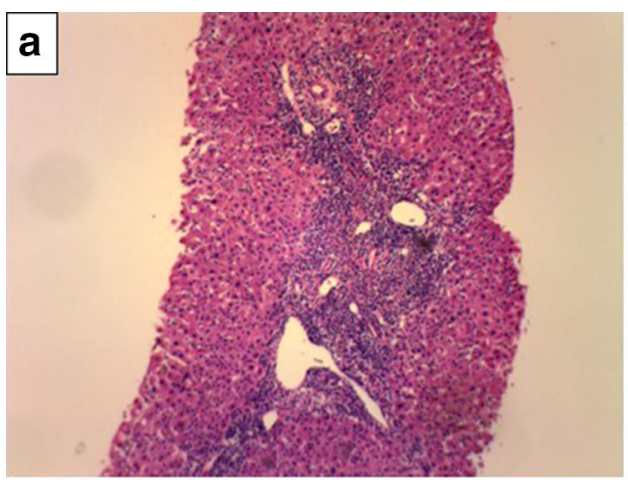
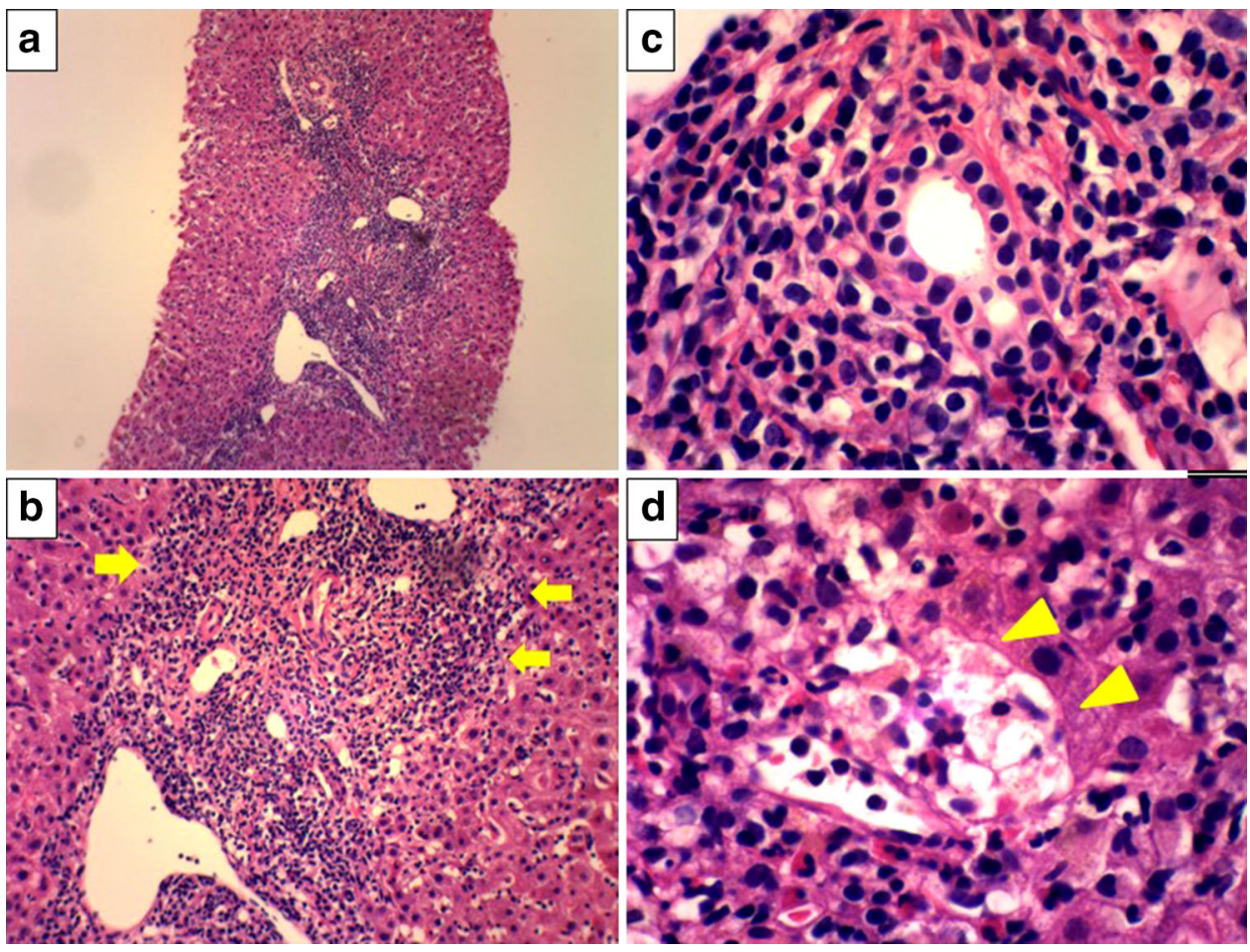

Fig. 2 Liver histology for case 2 (daughter). a Liver biopsy showing severe chronic hepatitis with marked interface hepatitis $(\times 2)$. b Magnified view of the liver biopsy showing dense lymphocytic/lymphoplasmacytic infiltrations in portal tracts and severe

period of time living with the mother for the first daughter (case 2) was same as that for the second daughter. The mother (case 1) was a housewife, which differed from both daughters' occupations as nurses. There were no histories of symptoms suggestive of viral infection.

piecemeal necrosis (arrow, $\times 5$ ). c Liver biopsy showing the absence of bile duct lesions, indicative of chronic nonsuppurative cholangitis and periductal granuloma $(\times 20)$. d Severe intralobular necrosis and degeneration of hepatocytes (arrowhead, $\times 20$ )

\section{Discussion}

We encountered two patients exhibiting overlapping clinical features of $\mathrm{PBC}$ and $\mathrm{AIH}$ in a family with a similar genetic background in terms of HLA-DRB1 haplotype. 
Fig. 3 Clinical course of case 2 (daughter). Liver biopsy was performed on the day of admission. Following diagnosis with an autoimmune liver disease with overlapping features of primary biliary cholangitis and autoimmune hepatitis, the patient was treated with a combination of $600 \mathrm{mg} /$ day ursodeoxycholic acid (UDCA) and $30 \mathrm{mg} /$ day prednisolone $(P S L)$ in accordance with the Japanese guidelines, resulting in an improvement of both clinical and biochemical findings within 1 month. Liver function has remained normal ever since, despite the subsequent tapering of PSL. Key: ANA antinuclear antibody, $A M A$ antimitochondrial antibody, $A M A$ $M 2$ anti-mitochondrial antibody-M2

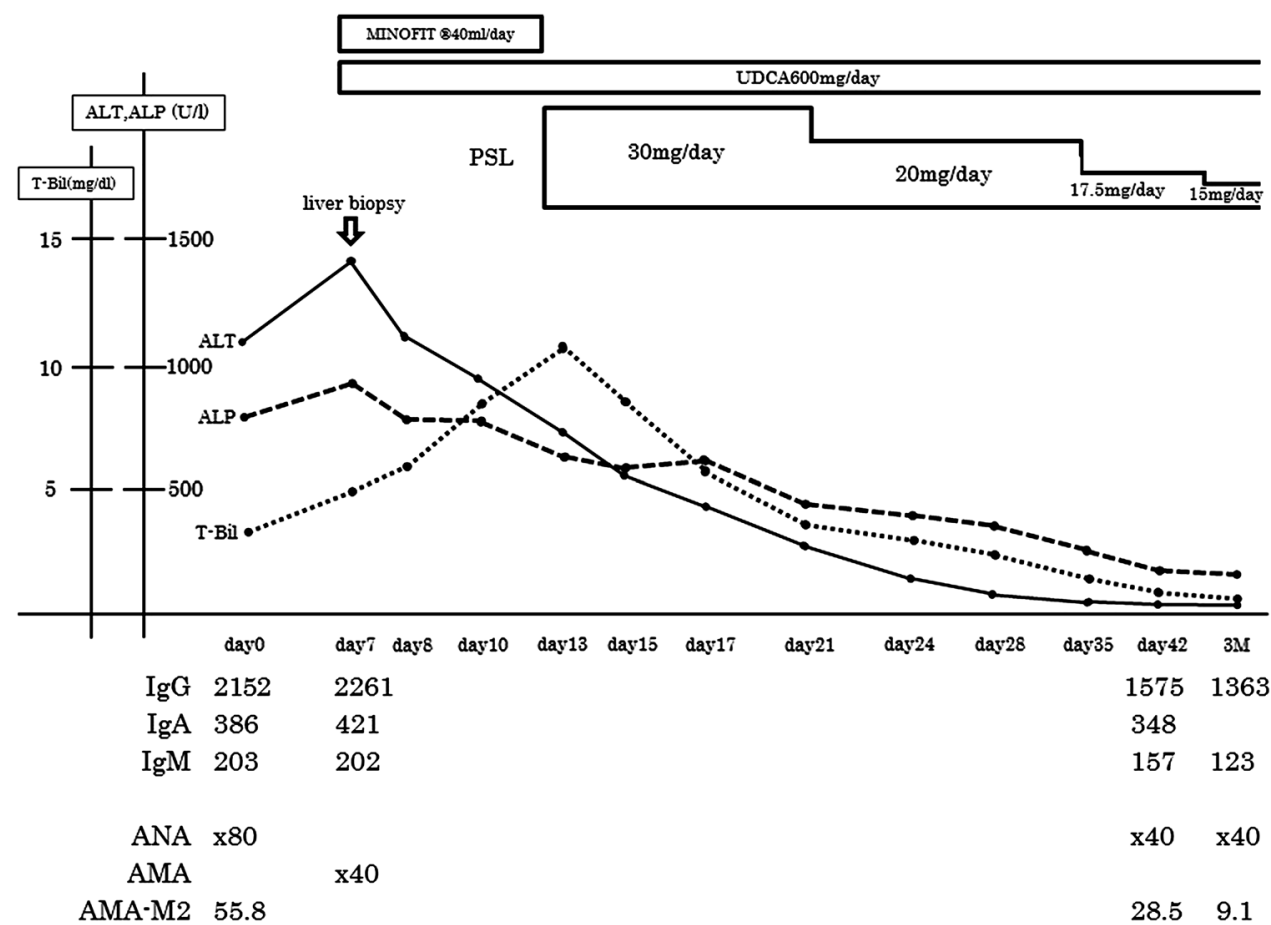

Autoimmune liver disease exhibiting features of both PBC and $\mathrm{AIH}$ may be present at diagnosis or develop during follow-up. At diagnosis, our cases already fell into two diagnostic criteria: the Paris criteria and Japanese guidelines $[4,8]$. In Japan, most patients with overlapping features of PBC and AIH have been reported to evolve into either $\mathrm{PBC}$ or AIH [5]. The development of AIH following liver transplantation for PBC has also been reported [10]. We suggest that our present cases provide further evidence of a link between these two diseases. Thus, autoimmune liver disease with overlapping features of $\mathrm{PBC}$ and $\mathrm{AIH}$, the socalled "PBC-AIH overlap syndrome," should no longer be considered as distinct diagnostic entities. However, PBCAIH overlap syndrome has been reported to progress rapidly to cirrhosis and liver failure [11]. In our present cases, the revised AIH scoring system implied a probable AIH. On the other hand, based on the simplified IAIHG scoring system, a probable AIH was implied for case 1, and a definite AIH for case 2. The simplified IAIHG scoring system is thought to be more effective in diagnostic specificity than the revised AIH scoring system [4]. The relevant scoring system should therefore be selected depending on the situation and when considering the possibility of rapid progression when features of PBC and AIH overlap. In the Japanese guidelines, it is recommended that physicians treat PBC-AIH overlap syndrome with PSL in combination with UDCA when PBC patients have also been described to develop a probable or definite AIH based on the simplified IAIHG scoring system [4, 6, 12]. In addition, our cases were diagnosed as definite AIH using the revised AIH score, which has previously been considered to exclude a diagnosis of probable AIH for other liver disorders [7]. Although PBC-AIH overlap syndrome may not be considered as a distinct diagnostic entity, the therapeutic strategy indicated by the Japanese guidelines is considered to be a realistic and useful clinical policy to treat patients with overlapping features of $\mathrm{PBC}$ and $\mathrm{AIH}$ in a swift and effective manner, even if they occur within the same family, as observed in our current cases. Although our cases responded to PSL therapy, it is necessary to be careful when patients exhibit a high ALP level, negative for ASMA and positive for gp210. This is because these indications represent the possible risk that patients will be nonresponsive to PSL therapy, as reported previously [13]. Furthermore, the long-term use of PSL can cause adverse effects, including hyperglycemia, osteoporosis, cataracts, weight gain, and an increased risk of opportunistic infections. When treating patients presenting with overlapping features of PBC and $\mathrm{AIH}$, physicians should be wary about such complications during follow-up. Fortunately, no adverse effects arose in either of our two patients as a result of the long-term use of PSL, although we will have to be careful with regard to the possibility of such complications arising over time. The current BMD levels in our two cases have remained higher than the normal range without alendronate treatment. However, the administration of alendronate may be necessary while monitoring BMD, as a previous study reported that alendronate led to the improvement of BMD in patients with PBC-related bone loss [14]. 
It has also been suggested that genetic susceptibility is an important factor in influencing the course of autoimmune liver disease and that HLA type is the most susceptible genetic factor responsible $[1,3]$. Although PBCAIH overlap syndrome in white individuals has been reported to be associated with positive tests for HLA-B8, DR3, or DR4, which are characterized as HLA haplotypes for AIH [2], both of our familial occurrence cases shared the same HLA haplotype, DRB1*04:05/DRB1*15:02. The second daughter's HLA haplotype, however, was DRB1*04:05/DRB $1 * 01: 01$. It is interesting to note that DRB $1 * 04: 05$ was the same within all family members, whereas the DRB $1 * 01: 01$ haplotype of the second daughter differed from the DRB $1 * 15: 02$ haplotype exhibited by both case 1 and case 2 . While it is well known that the frequency of the HLA-DRB1*04:05 haplotype is higher in Japanese AIH patients, it is difficult to accept that the HLADRB $1 * 04: 05$ haplotype is associated with the susceptibility to develop overlapping features of $\mathrm{PBC}$ and $\mathrm{AIH}$ within the same family. This is because the second daughter, with a HLA-DRB1*04:05 haplotype, has not developed overlapping features of $\mathrm{PBC}$ and $\mathrm{AIH}$ in spite of a positive test for the AMA-M2 antibody. Furthermore, overlapping features of PBC and AIH within the same family are very rare in proportion to the high frequency of HLA-DRB $1 * 04: 05$ in Japanese AIH patients. On the other hand, the association of DRB1*15:02, which differed from the second daughter's HLA haplotype, with autoimmune liver disease, including the overlapping features of PBC and AIH within the same family, remains unknown, although DR15 has been reported to be associated with other autoimmune diseases such as systemic lupus erythematosus (SLE) $[4,15]$. Earlier works have shown that SLE is associated with both AIH and PBC [16, 17]. Considering these conditions, it follows that the HLA-DRB1*15:02 haplotype may affect the susceptibility to develop overlapping features of PBC and AIH within the same family. None of our present family members had taken any medications to prevent such problems. Moreover, there are no means of predicting the onset of $\mathrm{PBC}$ and AIH overlap with respect to alcohol intake, smoking status, occupation, obstetric history, or viral serology. Although environmental conditions, the use of medications, and a variety of infectious factors were not considered to have influenced the onset of disease in either of our two patients, the possibility of their influences cannot be completely ruled out. As observed in our two patients, the exact mechanism underlying the onset of PBC and AIH overlap within the same family remains unclear. Therefore, it is very important to monitor the healthy second daughter closely as she was positive for the AMA-M2 antibody, as this might yield further knowledge with regard to what factors influence the onset of $\mathrm{PBC}$ and AIH overlap within the same family.
In case 2, pathological findings were compatible with $\mathrm{AIH}$, in that there were no pathological findings of $\mathrm{PBC}$, such as chronic nonsuppurative destructive cholangitis. Therefore, it was considered that AIH might represent a predominant pathophysiological factor in patients with overlapping features of $\mathrm{PBC}$ and $\mathrm{AIH}$ occurring within the same family, as observed in our cases. PBC patients with a genetic susceptibility to AIH may occasionally develop a mixed clinical picture of PBC and AIH. Existing reports also highlight that a genetic predisposition exists for both PBC and AIH, which share a common pathogenic mechanism [16].

The present cases suggest that a genetic predisposition is responsible for autoimmune liver disease with overlapping clinical features of PBC and AIH within the same family. It has also been reported that AIH develops among PBC patients after 6 months to 13 years [11]. As observed in our familial cases, the speed of sequential presentation of overlapping $\mathrm{PBC}$ and AIH features is very rapid. Consequently, autoimmune liver disease with clinical features of both PBC and AIH occurring within the same family should be treated very carefully by a combination of PSL and UDCA immediately, especially considering the possibility of rapid progression to liver cirrhosis and failure.

Acknowledgments The authors are grateful to Dr. Taro Takami, Institute for Department of Gastroenterology and Hepatology, Yamaguchi University Graduate School of Medicine, Japan, for his support in revising this manuscript.

\section{Complaince with ethical standards}

Conflict of interest The authors declare that they have no conflict of interest.

Human rights All procedures followed have been performed in accordance with the ethical standards laid down in the 1964 Declaration of Helsinki and its later amendments.

Informed consent Informed consent was obtained from all patients for being included in the study.

Open Access This article is distributed under the terms of the Creative Commons Attribution 4.0 International License (http://crea tivecommons.org/licenses/by/4.0/), which permits unrestricted use, distribution, and reproduction in any medium, provided you give appropriate credit to the original author(s) and the source, provide a link to the Creative Commons license, and indicate if changes were made.

\section{References}

1. Smyk D, Cholongitas E, Kriese S, et al. Primary biliary cirrhosis: family stories. Autoimmun Dis. 2011;2011:189585.

2. Lohse AW, zum Büschenfelde KH, Franz B, et al. Characterization of the overlap syndrome of primary biliary cirrhosis (PBC) and autoimmune hepatitis: evidence for it being a hepatic form of PBC in genetically individuals. Hepatology. 1999;29:1078-84. 
3. Abe M, Mashida T, Zeniya M, et al. Present status of autoimmune hepatitis in Japan: a nationwide survey. J Gastroenterol. 2011;46:1136-41.

4. Onji M, Zeniya M, Yamamoto K, et al. Autoimmnune hepatitis: diagnosis and treatment guide in Japan. Hepatol Res. 2014;44:368-70.

5. Boberg KM, Chapman RW, Hirschfield GM, et al. Overlap syndromes: the international autoimmune hepatitis group (IAIHG) position statement on a controversial issue. J Hepatol. 2011;54:374-85.

6. Hennes EM, Zeniya M, Czaja AJ, et al. Simplified criteria for diagnosis of autoimmune hepatitis. Hepatology. 2008;48:169-76.

7. Alvarez F, Berg PA, Bianchi FB, et al. International autoimmune hepatitis group report: review of criteria for diagnosis of autoimmune hepatitis. J Hepatol. 1999;31:929-38.

8. Kuiper EM, Zondervan PE, van Buuren HR. Paris criteria are effective in diagnosis of primary biliary cirrhosis and autoimmune hepatitis overlap syndrome. Clin Gastroenterol Hepatol. 2010;8:530-4.

9. Ishibashi H, Sakisaka S, Shimoda S, et al. Clinical guideline of primary biliary cirrhosis 2012. Kanzo. 2012;53:633-86 (in Japanese).

10. Jones DE, James OF, Portmann B, et al. Development of autoimmune hepatitis following liver transplantation for primary biliary cirrhosis. Hepatology. 1999;30:53-7.
11. Poupon R, Chazouilleres O, Corpechot C, et al. Development of autoimmune hepatitis in patient with typical primary biliary cirrhosis. Hepatology. 2006;44:85-90.

12. Tanaka A, Harada K, Ebinuma H, et al. Primary biliary cirrhosis-autoimmune hepatitis overlap syndrome: a rationale for corticosteroids use based on a nation-wide retrospective study in Japan. Hepatol Res. 2011;41:877-86.

13. Yoshida Y, Taniai M, Hashimoto E, et al. Clinical profile of primary biliary cirrhosis with features of autoimmune hepatitis: importance of corticosteroid therapy. Hepatol Res. 2014;44:947-55

14. Zein CO, Jorgensen RA, Clarke B, et al. Alendronate improves bone mineral density in primary biliary cirrhosis: a randomized placebo-control trial. Hepatology. 2005;42:762-71.

15. Niu Z, Zhang P, Tong Y. Value of HLA-DR genotype in systemic lupus erythematosus and lupus nephritis: a meta-analysis. Int $\mathrm{J}$ Rheum Dis. 2015;18:17-28.

16. Findor JA, Sorda JA, Daruich JR, et al. Familial association in autoimmune liver disease. Medicina. 2002;62:241-4.

17. Shizuma T. Coexistence of systemic lupus erythematosus and primary biliary cirrhosis. J Clin Cell Immunol. 2014;. doi:10. 4172/2155-9899.1000211. 